die ergriffenen sanitären Maßnahmen sondern noch auf andere Umstände zurückführen möchten.

Der Gedanke, daß es sich eventuell um eine Immunität in den Glossinen handeln körne, war ja naheliegend und suchten beide Autoren für diese Hypothese einen objektiven Beweis durch das Tierexperiment zu erbringen, der aber mißlang, so daß die Annahme einer Bildung von Antistoffen im Körper der Fliege nicht bestätigt werden konnte, und es muß wohl in den Trypanosomen selbst die Ursache für diese Verhältnisse gesucht werden.

Leopold Arzt (Wien).

Sutton, Richard. Mycetoma (MadurafuB) in Amerika. The Journal of the American Medical Association. 1913. Mai 3. p. 1339.

Wie Suttou hervorhebt, sind, bis auf eine alte Mitteilung $\mathrm{Ka}$ e m p fers erst in jüngster Zeit Fälle von Madurafuß aus Amerika beschrieben worden, fünfe im ganzen. Der Autor reiht zwei eigene Beobachtungen an. Fall I betrifft einen 24jährigen Arbeiter, bei dem die Krankheit sich vor 3 Jahren an eine Wunde der linken Fußsohle angeschlossen hatte. Dann kam es zu einer Geschwulstbildung, die trotz Operation an Größe zunahm. Wassermann reaktion und Pirquet negativ. Der affizierte Fuß ist geschwollen und mißgestaltet, mit Einbuchtungen versehen, aus denen sich eine eitrige Flüssigkeit ausdrïicken lieb. In diesem Material fanden sich Pilze, welche der ochroiden Form des Mycetoma glichen. Kulturversuche fielen negativ aus. Die Behandlung mit Jodkali, Kupfersulfat, Röntgenstrahlen führte nur zu einer geringen Besserung. Beim zweiten Falle wurde die Heilung durch Amputation erzielt. Der Verfasser berichtet eingehend über seine mikroskopischen Untersuchungen.

Fritz Juliusberg (Posen).

\title{
Sonstige Dermatosen.
}

Mörler, Apollo. Über Ekzeme bei Pferd und Rind. Diss. Bern 1912. Aus der Veterinärklinil Gießen (G meiner).

Bei den großen Haustieren kommen Ekzeme häufiger vor, als bisher angenommen wurde. Die Einteilung der Ekzemformen des Pferdes und Rindes, wie sie Friedberger und Fröhner vorgenommen haben, ist praktisch am zweckmäßigsten. Manche Ekzemformen des Pferdes sind klinisch von Sarkoptesräude schwer zu unterscheiden, besonders wenn sie generalierte Ausbreitung erlangt haben und Juckreiz besteht. Mikroskopische Untersuchung ist zwecks Stellung einer präzisen Diagnose unerläßlich. In ätiologischer Beziehung "kommen beim Pferde ausschließlich äußere Reize (chemischer, thermischer, mechanischer 
Natur) in Betracht. Beim Rinde sind nächstdem gewisse Schädlichkeiten durch Futterverhältnisse in Betracht zu ziehen. Kreolinliniment ist das sicherste Mittel gegen ausgebreitete und schwer zu bekämpfende Ekzeme. Bei chronischen und langanhaltenden, unheilbaren Ekzemen beteiligen sich so ziemlich alle Elemente der einzelnen Hautschichten, namentlich sind Drüsenatrophie, Hyperämie, Fibroblastenbildung und Vermehrung der bindegewebigen Elemente der Kutis die hauptsächlichsten histopathologischen Befunde. Weiler (Leipzig).

Devoto, L. Ätiologie und Klinik der Pellagra. Wiener med. Wochenschr. 1913. Nr. 1.

Nach dem Stande unserer Kenntnisse kann man die Pellagra als eine schleichende chronische Intoxikation ansehen, ausgezeichnet durch frühzeitig einsetzende Veränderungen des Nervensystems und durch ein großes Heer von Erscheinungen, welche auf Störungen verschiedener Organe, auch der Nebenniere, hinweisen. Es fehlen selten die Hautveränderungen, die unter dem Einflusse des Sonnenlichtes sich einstellen. Die Pellagra ist in ihrem Beginne eine benigne Erkrankung.

\section{Viktor Bandler (Prag).}

Mills, H. P. Pellagra. Pathologie des GastroIntestinaltraktus. The Journal of the American Medical Association. 1913. März 22. p. 889.

Mills berichtet über vier Fälle von Pellagra, wobei der Hauptwert auf die Beschreibung der Magen-Darm-Veränderungen gelegt ist. In allgemeinen fanden sich die Erscheinungen chronischer katarrbalischer Entzündung, aber an einigen Stellen waren die Veränderungen stärker und entsprachen der von Coplin beschriebenen hämorrhagischen Entzündung der Schleimhäute. Fritz Juliusberg (Posen).

Dearman, W. A. Die Ätiologie der Pellagra.

Gray, J. W. Die Behandlung der Pellagra mit Opium. Mississippi State Medical Association. 1913. April 7. The Journal of the American Medical Association. 1913. Mai 10. p. $1485 / 1486$.

Dearman machte einen Affen pellagrakrank, indem er ihn mit Pseudomembranen der Zunge und mit Speichel von Pellagrakranken fütterte. Er starb mit den Symptomen der Pellagra. Er hält die Pellagra für eine gastrointestinale Intoxikation durch einen spezifischen Saprophyten, der auf Kohlenhydraten wächst und ein eigenartiges Toxin bildet. Gray behandelt die Pellagra mit Erfolg mit Opium.

$$
\text { Fritz Juli usberg (Posen). }
$$

Grimm, R. M. Pellagra: einige Tatsachen über ihre Epidemiologie. The Journal of the American Medic. Association. 1913. Mai 10. p. 1423. 
Grimm kommt in seinen Ausführungen, die auf Beobachtungen von 323 Fällen von Pellagra basieren, zu folgenden Schlüssen: Es entwickeln sich mehr Fälle unter den Weißen als unter den Negern. Es kommen bei beiden Rassen mehr Fälle unter den Frauen vor. Die meisten Fälle entstanden im Alter von 20-40 Jahren. Unter den verheirateten und verwitweten Pellagrakranken prädominieren die Frauen. Die meisten Fälle traten während der Monate Mai und Juni auf. Die meisten Fälle entstanden in der Umgebung anderer Fälle. Nichts spricht dafür, daß die Pellagra hereditär ist.

$$
\text { Fritz Juliusberg (Posen). }
$$

Langmead, Frederick. Ein Fall von Addisonscher Krankheit bei einem $10 \mathrm{jährigen} \mathrm{Knaben.} \mathrm{The} \mathrm{Lan-}$ cet. 1913. Februar 15. p. 449.

Langmead berichtet ausführlich über den seltenen Fall von Ad d is on scher Krankheit bei einem Kinde. Die Arbeit enthält ein eingehendes Sektionsprotokoll.

$$
\text { Fritz Juliusberg (Posen). }
$$

Saisawa, K. Beitrag zur Ätiologie des Erythema exsudatirum multiforme. Deutsche med. Wochenschrift. Nr. 17. 1913.

Beim Erythema exsudativum multiforme fand Saisawa einen Bazillus, dessen Züchtung zwar nicht gelang, der aber füı die Erkrankung pathogen zu sein schien. Der Verlauf der Krankheit zeigte bei dem jungen, stets gesunden Patienten das Bild einer akuten Infektion: Fieberhaftes Vorstadium, schnelle Verbreitung der Erytheme über den ganzen Körper, unter zunehmendem Fieber und Schwächezustand Nephritis, Vergrößerung von Leber und Milz, Herzerweiterung, Lungenödem. Als unter starkem Schweißausbruch auch die Urinmenge zunahm, besserte sich der Befund der inneren Organe schnell, die Kraft kehrte wieder, die Erytheme blaßten ab. Während der Krankheit fanden sich in Blut und Harn stäbchenförmige Mikroben, welche während der Genesung verschwanden, um vermehrten Leukozyten Platz zu machen, bis nach einigen Wochen der normale Zustand zurückkehrte. Max Josep h (Berlin).

Geber, János. Ein Fall von septis chem Erythem. Budapesti Orvosi Ujság. 1913. Nr. 23.

Bei einem Kranken trat nach einer Halsentzündung in Begleitung von Schüttelfrost ein dem Erythema exsudativum multiforme ähnliches Erythem auf, welches sich im Verlaufe von 3 Wochen öfters wiederholte. Die Ursache der Krankheit ist wahrscheinlich in den Blutkreislauf getretenen Bakterien zu suchen, obzwar die Blutuntersuchung auf Bakterien negativ war. Alfred Roth (Budapest). 